\title{
Producción científica latinoamericana indexada en Scopus en el área de las ciencias agropecuarias: análisis del período 1996-2016
}

\author{
Scientific production in Latin American indexed in Scopus in \\ farming sciences: an analysis during the period 1996-2016 \\ José Guerrero-Casado ${ }^{1 *}$
}

\begin{abstract}
RESUMEN
El objetivo de este trabajo fue realizar un análisis descriptivo y explicativo del número de artículos publicados por los países de América Latina y el Caribe en Scopus dentro de las categorías Agricultura y Ciencias Biológicas, y Veterinaria para el período 1996-2016. Los países con más artículos publicados fueron Brasil, México, Argentina y Chile; sin embargo, Uruguay fue el país con más artículos publicados por cada millón de habitantes. Se obtuvo una relación positiva entre el número de artículos publicados por millón de habitantes en cada país y su renta per cápita, el porcentaje del producto interno bruto (PIB) destinado a investigación y desarrollo (I+D) y el número de investigadores por millón de habitantes. Además, los países con mayor porcentaje de población rural y donde la agricultura aporta más al PIB tuvieron menos producción científica, debido a que ambas variables están negativamente relacionadas con la renta per cápita. Por lo tanto, se concluye que los países con más artículos en esta área de conocimiento son aquellos con mayores recursos económicos, y no aquellos donde las actividades agropecuarias tienen mayor importancia.

Palabras clave: cienciometría, ciencias agropecuarias, Latinoamérica, producción científica, Scimago.
\end{abstract}

\section{ABSTRACT}

The goal of this paper was to carry out a descriptive and explicative analysis of the number of scientific papers published by the countries of Latin America and Caribbean indexed in Scopus within the areas Agriculture and Biological Sciences, and Veterinary during the period 1996-2016. The countries with more articles published were Brazil, Mexico, Argentina and Chile, although Uruguay was the county with more articles published per million of habitants. A positive relationship was found between the number of articles published per million of habitants and the per capita income, the percentage of the Gross Domestic Product (GDP) devoted to research and development $(R+D)$, and the number of researcher per million of habitants. Moreover, the countries with higher percentage of rural population in which the agriculture contribute more to the GDP had less articles, probably because both variables are negative associated with the GDP. Therefore, we concluded that the countries with better economic resources published more articles in this field, and not those in which the farming activities are more important.

Key words: farming sciences, Latin America, scientific production, Scimago, Scopus.

\section{Introducción}

Latinoamérica es una región con un gran desequilibrio político, social y económico. Sin embargo, en general, la mayoría de los países de esta región sufren de los mismos problemas que hacen que no sea una de las regiones con mayor producción científica (Santa and Herrero Solana, 2010). Entre estos problemas comunes podemos destacar la baja inversión en I+D, escasa inversión privada en actividades científico-tecnológicas, un escaso número de profesionales dedicados a la investigación y el desarrollo tecnológico, o el mayor costo de los materiales y equipamientos científicos (Zenteno-Savín et al., 2007; Santa and Herrero Solana, 2010; Bonilla et al., 2015; Chinchilla-Rodríguez et al., 2015). Por ejemplo, según datos de la Red de Indicadores de Ciencia y Tecnología-Iberoamericana e Interamericana (RICYT 2017), para el año 2015 la inversión en

\footnotetext{
1 Facultad de Ciencias Veterinarias, Universidad Técnica de Manabí. Portoviejo, Manabí (Ecuador).

* Autor por correspondencia: guerrero.casado@gmail.com
} 
I+D en la región fue de $0,75 \%$ sobre el Producto Interno Bruto, mientras que en Estados Unidos la cifra fue de $2,79 \%$ y en los países de la Unión Europea del 2,05\% (World Bank Group, 2017).

Por estos motivos, América Latina a pesar de ser un área muy extensa que abarca 46 países, tiene un peso relativamente bajo en la producción científica mundial, así como un impacto (citas por documento) bajo en comparación con las regiones más desarrolladas (Hermes-Lima et al., 2007). Sin embargo, es reseñable el aumento que ha experimentado la región en el número de artículos publicados en los últimos años. Según datos de RICYT (2017), en América Latina y el Caribe se publicaron 22.138 artículos en Scopus en el año 1996, mientras que está cifra aumentó a 126.620 en el año 2015, lo que supone un aumento de 5,72 puntos, mucho más que por ejemplo el aumento experimentado en Estados Unidos $(1,82)$ o Canadá (2,31).

Varios trabajos bibliométricos han analizado la producción científica latinoamericana incluida en Scopus en distintas categorías por medio de la herramienta Scimago Journal \& Country Rank (http://www.scimagojr.com/) (ChinchillaRodríguez et al., 2015; Bonilla et al., 2015). Estos trabajos han demostrado que el PIB, la renta per cápita o el número de investigadores explican las diferencias observadas entre países en cuanto a la producción científica. Sin embargo, ningún trabajo ha analizado la producción científica en Scopus en las ciencias agropecuarias. Latinoamérica es una región donde la producción primaria tiene una gran importancia, debido a que en numerosos países el sector industrial aún no está muy desarrollado. Se estima que los países de la región contribuyen con el $11 \%$ del valor de la producción alimentaria mundial y poseen el $24 \%$ de la tierra cultivable del mundo (Banco Interamericano de Desarrollo, 2017), además de producir un poco más del $23 \%$ de la carne bovina y de búfalo, y el 21,40\% de la carne de ave a nivel mundial (FAO, 2017).

Por estos motivos, resulta de interés estudiar la producción científica en el área de las ciencias agropecuarias, e intentar explicar las diferencias observadas entre países mediante variables económicas y variables que caractericen la importancia del sector agropecuario en cada país. La hipótesis de partida es que la producción científica de un país estará relacionada con la inversión realizada en investigación así como con la importancia del sector agropecuario. En teoría, mientras mayor peso tenga la agricultura y ganadería en la economía del país, mayor importancia debería tener la investigación realizada en este sector y, por tanto, deberían tener más publicaciones que otros países donde el sector agropecuario es menos importante.

Para ello, en este trabajo se analizan distintos parámetros de la producción científica en Scopus durante el período 1996-2016. Concretamente, los objetivos este trabajo fueron: 1) describir la evolución de las publicaciones en Scopus durante el período 1996-2016; 2) describir cuáles fueron los países más productivos; y 3) relacionar el número de artículos publicados en cada país con variables económicas y variables de tipo agropecuario.

\section{Materiales y Métodos}

\section{Datos de producción científica}

Los datos de artículos publicados por país fueron obtenidos de la web de Scimago Journal \& Country Rank (http://www.scimagojr.com/), herramienta elaborado por SCImago Research Group que ofrece indicadores de revistas y países a partir de datos recopilados de Scopus. En primer lugar, se obtuvo un fichero que contenía los artículos publicados por cada país de la región de Latinoamérica y el Caribe para el período 1996-2016 en el área de Agricultura y Ciencias Biológicas (subject area Agriculture and Biological Sciences), y otro para el área de Veterinaria (subject area Veterinary). Además para conocer la evolución en el número de artículos publicados por año en cada país, se descargaron los datos de publicaciones en cada año para las dos áreas consideradas de forma independiente. Para el análisis descriptivo (medias y tendencias) se utilizaron todos los países latinoamericanos con artículos publicados en Scimago $(\mathrm{n}=28)$, mientras que para el análisis estadístico se descartaron aquellos países con menos de un millón de habitantes $(n=23)$.

\section{Variables utilizadas}

Para cada país, se obtuvieron una serie de variables explicativas económicas y agropecuarias, las que fueron obtenidas del portal World Bank (http://www.worldbank.org/) (World Bank Group 2017) y del portal UNESCO's Institute of Statistics 
(http://uis.unesco.org/) (UNESCO, 2017). Estas bases de datos ya han sido previamente utilizadas en trabajos bibliométricos (Chinchilla-Rodríguez et al., 2015). Las variables seleccionadas de tipo económico fueron la renta per cápita, la inversión en I+D, la inversión en educación, y el número de investigadores por millón de habitantes, las que han sido descritas previamente como variables que afectan a la productividad científica (Holmgren et al., 2004; King, 2004; Hermes-Lima et al., 2007; Chinchilla-Rodríguez et al., 2015). Por otra parte, se seleccionaron una serie de variables que caracterizan la importancia del sector agropecuario en cada país, las cuales fueron: el porcentaje de tierra dedicada al cultivo, el aporte de las actividades agropecuarias al PIB, el porcentaje de la población que vive en zonas rurales y la producción ganadera (Tabla 1). Para todas las variables, se calculó el valor medio comprendido EN el período 1996-2016.

\section{Análisis estadístico}

En primer lugar, para estandarizar el número de publicaciones según la población del país, se calculó el número de artículos publicados por millón de habitantes (Chinchilla-Rodríguez et al., 2015) para el área de Agricultura y Ciencias Biológicas, y para el área de Veterinaria de forma independiente. Posteriormente, debido a que ambas variables no seguían una distribución normal, se realizaron análisis de correlación de Spearman para comprobar la relación entre el número de documentos por millón de habitantes y las variables explicativas (renta per cápita, inversión en I+D, inversión en educación, número de investigadores, porcentaje de tierra cultivada, aporte de las actividades agropecuarias al PIB, porcentaje de población rural y producción ganadera).

En segundo lugar, se utilizó el número citas por documento en cada país como indicador de la calidad de las publicaciones (Hermes-Lima et al., 2007). Esta variable tampoco siguió una distribución normal y, por lo tanto, se realizaron test de Spearman para comprobar la correlación entre el número de citas por documento y las variables explicativas. Finalmente, se realizó otro análisis de correlación entre el número de documentos por millón de habitantes y el número de citas por documento en ambas áreas.

\section{Resultados y Discusión}

\section{Producción en el campo Agricultura y Ciencias Biológicas}

En total, durante el período 1996-2016 se publicaron 277.673 artículos, lo que supone un $7,75 \%$ de la producción mundial. 3.845 documentos

Tabla 1. Descripción de las variables utilizadas en este estudio.

\begin{tabular}{|c|c|}
\hline Variable & Descripción \\
\hline Documentos/millón de habitantes & (Total de artículos publicados / población del país)*1000000 \\
\hline Citas por documento & $\mathrm{N}^{\circ}$ total de citas / $\mathrm{N}^{\circ}$ total de documentos \\
\hline Renta per cápita & $\begin{array}{l}\text { Valor en USD (\$) resultante de dividir el producto interno bruto entre la población } \\
\text { a mitad de año. }\end{array}$ \\
\hline Inversión en investigación y desarrollo (I+D) & $\begin{array}{l}\text { Porcentaje del PIB (\%) que es destinado a realizar investigación básica y aplicada, } \\
\text { y el desarrollo experimental. }\end{array}$ \\
\hline Inversión en educación & Porcentaje (\%) del gasto total del gobierno dedicado a la educación. \\
\hline $\mathrm{N}^{\mathrm{o}}$ de investigadores & $\begin{array}{l}\text { Número de personas dedicadas a la investigación y el desarrollo por cada millón } \\
\text { de habitantes. }\end{array}$ \\
\hline Porcentaje de tierra cultivada & $\begin{array}{l}\text { Porcentaje del territorio del país dedicado (\%) a la agricultura, bien sea cultivos } \\
\text { permanentes o temporales. }\end{array}$ \\
\hline Aporte de las actividades agropecuarias al PIB & Porcentaje del PIB (\%) aportado por las actividades ganaderas, agrícolas y forestales. \\
\hline Porcentaje de población rural & Porcentaje de la población (\%) que vive en zonas rurales. \\
\hline Producción ganadera $(2004-2006=100)$ & $\begin{array}{l}\text { El índice de producción ganadera incluye carne y leche de todas las fuentes, } \\
\text { productos lácteos, huevos, miel, lana, cueros y pieles. }\end{array}$ \\
\hline
\end{tabular}


fueron publicados en 1996, cifra que aumentó a 26.357 en 2016, lo que supone un aumento de 6,85 puntos. El promedio fue de 13.324 artículos publicados por año. En la Tabla 2 se muestran los datos de los 10 países más productivos en el área de Agricultura y Ciencias Biológicas según el número de artículos publicados. Brasil, Argentina, México y Chile fueron los países con mayor número de publicaciones, y por el contrario, El Salvador, República Dominicana, Paraguay y Haití fueron los países con menos artículos publicados, con menos de 200 artículos durante el período 19962016. Sin embargo, Uruguay y Chile fueron los países con más artículos publicados por millón de habitantes.

Los resultados del coeficiente de correlación de Spearman mostraron una relación positiva y estadísticamente significativa entre el número artículos publicados por millón de habitantes en cada país y la renta per cápita, el número de investigadores y la inversión en I+D (Tabla 3). Por otra parte, el número de publicaciones estuvo negativamente relacionado con el porcentaje de población rural y la contribución de las actividades agropecuarias al PIB. No se encontró ninguna relación significativa entre el número de citas por documento y las variables explicativas (Tabla 3 ). Por último, tampoco hubo una relación entre el número de documentos por millón de habitantes y las citas por documento $(\rho=0,28 ; p=0,1819)$.

\section{Producción en el campo de Veterinaria}

En el área de Veterinaria, en Latinoamérica y el Caribe se publicaron 38.221 artículos durante el período 1996-2016, lo que equivale a un 9,73\% de los artículos publicados a nivel mundial. En 1996 se publicaron 458 , y 3.173 en 2016 , lo que supone

Tabla 2. Producción científica de los 10 países más productivos de Latinoamérica y el Caribe según el número de artículos publicados en el área Agricultura y Ciencias Biológicas durante el período 1996-2016.

\begin{tabular}{lrrrrcc}
\hline \multicolumn{1}{c}{ País } & Docs. & Docs. citados & \multicolumn{1}{c}{ Citas } & Citas / docs. & Índice $\mathrm{H}$ & Docs. millón habitantes \\
\hline Brasil & 133.999 & 131.627 & 1.226 .705 & 9,15 & 200 & 644,7 \\
México & 41.812 & 41.152 & 517.185 & 12,37 & 167 & 329,1 \\
Argentina & 36.870 & 36.262 & 491.064 & 13,32 & 164 & 849,2 \\
Chile & 18.038 & 17.699 & 228.598 & 12,67 & 126 & 100,5 \\
Colombia & 11.345 & 11.047 & 104.333 & 9,2 & 107 & 235,2 \\
Venezuela & 5.988 & 5.912 & 61.470 & 10,27 & 92 & 192,4 \\
Cuba & 4.139 & 4.069 & 30.996 & 7,49 & 70 & 363,4 \\
Costa Rica & 4.065 & 3.986 & 56.412 & 13,88 & 91 & 845,4 \\
Perú & 3.637 & 3.544 & 51.022 & 14,03 & 88 & 115,9 \\
Uruguay & 3.588 & 3.527 & 51.331 & 14,31 & 79 & 104,5 \\
Media & 9.916 & 9.738 & 110.269 & 14,22 & 70,64 & 518,7 \\
\pm DS & 26.448 & 25.986 & 255.562 & 5,72 & 50,5 & 890 \\
\hline
\end{tabular}

La última fila muestra la media para cada parámetro considerando todos los países $(n=23)$. DS = desviación estándar.

Tabla 3. Coeficiente de correlación de Spearman ( $\rho$ ) entre el número de documentos por millón de habitantes o las citas por documento y las distintas variables explicativas seleccionadas en el área de Agricultura y Ciencias Biológicas.

\begin{tabular}{lcl}
\hline & Doc. por millón habitantes & Citas por documento \\
\hline Renta per cápita & $\rho=0,85 ; \mathrm{p}=0,0001$ & $\rho=0,15 ; \mathrm{p}=0,4697$ \\
Inversión en I+D** & $\rho=0,74 ; \mathrm{p}=0,0010$ & $\rho=0,42 ; \mathrm{p}=0,0505$ \\
Inversión en educación & $\rho=0,04 ; \mathrm{p}=0,8675$ & $\rho=0,32 ; \mathrm{p}=0,0605$ \\
$\mathrm{~N}^{\circ}$ de investigadores* & $\rho=0,66 ; \mathrm{p}=0,005$ & $\rho=-0,21 ; \mathrm{p}=0,3678$ \\
Porcentaje de tierra cultivada & $\rho=-0,25 ; \mathrm{p}=0,2447$ & $\rho=-0,21 ; \mathrm{p}=0,3213$ \\
Aporte de las actividades agropecuarias al PIB & $\rho=-0,56 ; \mathrm{p}=0,0105$ & $\rho=0,06 ; \mathrm{p}=0,7938$ \\
Porcentaje de población rural & $\rho=-0,6 ; \mathrm{p}=0,005$ & $\rho=-0,11 ; \mathrm{p}=0,6069$ \\
Producción ganadera (2004-2006=100) & $\rho=0,19 ; \mathrm{p}=0,3637$ & $\rho=0,10 ; \mathrm{p}=0,6463$ \\
\hline
\end{tabular}

* El tamaño de muestra para el número de investigadores por país fue 19. **El tamaño de muestra para la inversión en I+D fue 21 
un aumento de 6,93 puntos (Tabla 4; Figura 1), con un valor medio de 1.820 documentos por año. Brasil, México, Argentina y Chile también fueron los países con mayor número de artículos, destacando Uruguay y Trinidad \& Tobago en el número de artículos publicados por habitante. Por el contrario, Haití, El Salvador, Honduras y la República Dominicana fueron los países con menor número de artículos publicados $(<20)$.

El número de artículos publicados mostró una relación positiva y significativa con la renta per cápita, la inversión en I+D, y el número de investigadores, mientras que un relación negativa y significativa se encontró con el aporte de la agricultura al PIB y el porcentaje de población rural (Tabla 5). Tampoco se encontró ninguna relación significativa entre el número de citas por documento y las variables explicativas (Tabla 5), ni entre el número de documentos por millón de habitantes y las citas por documento $(\rho=-0,08 ; p=0,643)$.

Este trabajo muestra como la producción científica en Scimago dentro del área de las ciencias agropecuarias ha aumentado significativamente en los últimos años en Latinoamérica, particularmente a partir del 2005 (Figuras 1). Este incremento también ha sido registrado en otras áreas del conocimiento, debido principalmente al crecimiento económico registrado en los países latinoamericanos durante los últimos años, un cambio en las políticas de investigación, una mayor colaboración internacional y un aumento del número de revistas locales indexadas en las bases internacionales (De Moya-Anegón and Herrero-Solana, 1999;

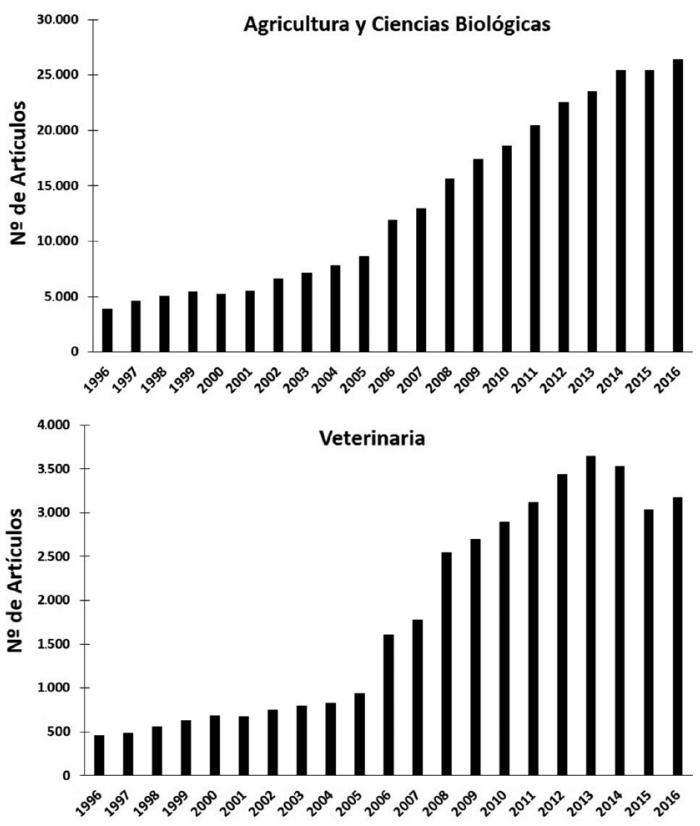

Figuta 1. Evolución del número de artículos publicados en el área de la Agricultura y Ciencias Biológicas, y Veterinaria por autores con afiliación de alguna institución de Latinoamérica y el Caribe.

Aguado-López et al., 2012; Collazo-Reyes, 2014; Bonilla et al., 2015; Aguado-López et al., 2016). Sin embargo, existe una importante brecha entre los países latinoamericanos. En general, Brasil, Argentina, Chile y México son los países con más artículos publicados (todas las categorías incluidas; consultado en http://www.scimagojr.com/ countryrank.php?region=Latin\%20America), hecho

Tabla 4. Producción científica de los 10 países más productivos de Latinoamérica y el Caribe según el número de artículos publicados en el área Veterinaria durante el período 1996-2016.

\begin{tabular}{|c|c|c|c|c|c|c|}
\hline País & Docs. & Docs. citados & Citas & Citas / doc. & Índice $\mathrm{H}$ & Docs. millón habitantes \\
\hline Brasil & 24.141 & 23.748 & 150.132 & 6,22 & 86 & 116,1 \\
\hline México & 3.773 & 3.729 & 27.947 & 7,41 & 54 & 29,7 \\
\hline Argentina & 3.095 & 3.040 & 32.948 & 10,65 & 60 & 71,3 \\
\hline Chile & 1.389 & 1.376 & 9.527 & 6,86 & 34 & 77,4 \\
\hline Colombia & 1.315 & 1.288 & 8.818 & 6,71 & 41 & 27,26 \\
\hline Venezuela & 1.288 & 1.262 & 6.144 & 4,77 & 33 & 41,4 \\
\hline Cuba & 689 & 669 & 4.180 & 6,07 & 30 & 60,5 \\
\hline Perú & 643 & 633 & 3.427 & 5,33 & 29 & 20,5 \\
\hline Uruguay & 531 & 522 & 5.681 & 10,7 & 33 & 154,7 \\
\hline Tri. \& Tobago & 212 & 197 & 1.827 & 8,62 & 22 & 155,9 \\
\hline Media & 1.644 & 1.617 & 11.327 & 9,67 & 25,4 & 38,3 \\
\hline$\pm \mathrm{DS}$ & 5.004 & 4.923 & 31.438 & 5,19 & 20,2 & 47,4 \\
\hline
\end{tabular}

La última fila muestra la media para cada parámetro considerando todos los países $(n=23)$. DS = desviación estándar. 
Tabla 5. Coeficiente de correlación de Spearman $(\rho)$ entre el número de documentos por millón de habitantes o las citas por documento y las distintas variables explicativas seleccionadas en el área de Veterinaria.

\begin{tabular}{lcl}
\hline & Doc. por millón habitantes & Citas por documento \\
\hline Renta per cápita & $\rho=0,80 ; \mathrm{p}=0,0002$ & $\rho=0,10 ; \mathrm{p}=0,6430$ \\
Inversión en I+D** & $\rho=0,72 ; \mathrm{p}=0,0014$ & $\rho=-0,05 ; \mathrm{p}=0,8344$ \\
Inversión en educación & $\rho=-0,18 ; \mathrm{p}=0,3963$ & $\rho=0,31 ; \mathrm{p}=0,239$ \\
$\mathrm{~N}^{\mathrm{o}}$ de investigadores* & $\rho=0,78 ; \mathrm{p}=0,0009$ & $\rho=-0,05 ; \mathrm{p}=0,8233$ \\
Porcentaje de tierra cultivada & $\rho=-0,18 ; \mathrm{p}=0,3963$ & $\rho=0,02 ; \mathrm{p}=0,9188$ \\
Aporte de las actividades agropecuarias al PIB & $\mathrm{r}=-0,61 ; \mathrm{p}=0,0048$ & $\rho=0,02 ; \mathrm{p}=0,9188$ \\
Porcentaje de población rural & $\mathrm{r}=-0,59 ; \mathrm{p}=0,0053$ & $\rho=0,03 ; \mathrm{p}=0,8711$ \\
Producción ganadera (2004-2006=100) & $\mathrm{r}=0,15 ; \mathrm{p}=0,4754$ & $\rho=-0,04 ; \mathrm{p}=0,8348$ \\
\hline
\end{tabular}

*El tamaño de muestra para el número de investigadores por país fue 19. **El tamaño de muestra para la inversión en I+D fue 21 .

que se repite para las categorías de las ciencias agropecuarias, y que es en parte explicado por ser países más grandes y poblados. Sin embargo, al estandarizar por el número de habitantes, destaca la productividad de Uruguay y Chile.

A pesar de que la baja producción de Latinoamérica en el contexto mundial es atribuida a una baja inversión en I+D y a el escaso número de investigadores, estos parámetros son útiles para explicar la diferencia observada entre países latinoamericanos en cuanto a la producción científica, ya que hay una cierta variabilidad entre países en cuanto al número de publicaciones y la inversión realizada en investigación (Holmgren et al., 2004; Aguado-López et al., 2014). Nuestros resultados sugieren que dicha brecha es explicada por desigualdades económicas (renta per cápita) y por la inversión que los países realizan investigación y desarrollo tecnológico. En otras palabras, aquellos países con mayor renta per cápita, mayor inversión en I+D y mayor número de investigadores son los que publican más artículos científicos en el campo de las ciencias agropecuarias.

La hipótesis de partida de este estudio era que la producción científica sería mayor en aquellos países donde las actividades agropecuarias son más relevantes. Sin embargo, la producción científica no fue explicada por las variables de carácter agropecuario seleccionadas. Además, los países donde la agricultura aporta más al PIB y donde hay más población rural (que usualmente depende de la agricultura y la ganadería) la producción científica fue menor. Esto podrá ser explicado por la relación negativa que existe entre la renta per cápita y el aporte de la agricultura al PIB $(r=-0,77 ; p=0,0005)$ y el porcentaje de población rural $(\mathrm{r}=-0,76 ; \mathrm{p}=0,0004)$.
Esto sugiere que los países donde las actividades agropecuarias son más importantes realizan menos inversión en investigación en esta área debido a su menor poder económico, lo que también afecta a su producción científica y, por ende, podría limitar el correcto desarrollo del sector.

Curiosamente ninguna de las variables seleccionadas explica la variación en el número de citas por documento entre países. El número de citas que cada país recibe por documento publicado en las revistas incluidas en Scopus podría ser un indicativo del impacto y la visibilidad de los trabajos publicados (Tahamtan et al., 2016), lo que a priori también podría estar influenciado por las variables económicas. Sin embargo, nuestros resultados no muestran ningún resultado estadísticamente significativo con variables de tipo económico o agropecuario. Es conocido que los países más desarrollados producen artículos de mayor impacto que los países en vías de desarrollo (King, 2004; Holmgren et al., 2004; Hermes-Lima et al., 2007); sin embargo, para el caso de las ciencias agropecuarias en Latinoamérica parece no existir una relación entre la renta per cápita o la inversión en I+D con el impacto que alcanzan sus publicaciones. Además, no existió una relación entre el número de trabajos publicados por país y el número de citas por documento, lo que significa que una mayor producción científica no implica un mayor impacto de las publicaciones en Latinoamérica para el área de las ciencias agropecuarias.

\section{Conclusiones}

En resumen, el análisis realizado en este trabajo certifica, por un lado, el aumento experimentado 
en Latinoamérica y el Caribe en las publicaciones indexadas en Scopus durante el período 19962016. Sin embargo, existen grandes diferencias en cuanto a la producción científica entre los países de la región, lo que es parcialmente explicado por la renta per cápita, la inversión en I+D y el número de investigadores, y no por la importancia de las actividades agropecuarias en la economía del país en cuestión. De hecho, los países que más dependen de la agricultura y la ganadería (medido como su aportación al PIB y por el porcentaje de población rural) mostraron menos publicaciones en la base de datos Scopus. El menor número de publicaciones (derivadas de las investigaciones) podría frenar el desarrollo del sector productivo en los países donde las actividades agropecuarias son más importantes.

\section{Literatura Citada}

Aguado-López, E.; Becerril-García, A. and Becerril-García, A. 2016. ¿Publicar o perecer? El caso de las Ciencias Sociales y las Humanidades en Latinoamérica. Revista Española de Documentación Científica 9(4): e151. doi: http://dx.doi. org/10.3989/redc.2016.4.1356

Aguado-López, E.; Becerril-García, A.; Leal Arriola, M. and Martínez-Domínguez, N.D.

2014. Iberoamérica en la ciencia de corriente principal (Thomson Reuters/Scopus): una región fragmentada. Interciencia 39: 570-579.

Aguado-López, E.; Adolfo Garduño-Oropeza, G.; Rogel-Salazar, R., and Fernanda Zúñiga-Roca, M.

2012. The need and viability of a mediation index in Latin American scientific production and publication. Aslib Proceedings 64, 8-31.

Bonilla, C.A.; Merigó, J.M. and Torres-Abad, C.

2015. Economics in Latin America: a bibliometric analysis. Scientometrics 105, 1239-1252.

Chinchilla-Rodríguez, Z.; Zacca-González, G.; Vargas-Quesada,

B. and Moya-Anegón, F.

2015. Latin American scientific output in Public Health: combined analysis using bibliometric, socioeconomic and health indicators. Scientometrics 102, 609-628.

Collazo-Reyes, F.

2014. Growth of the number of indexed journals of Latin America and the Caribbean: the effect on the impact of each country. Scientometrics 98, 197-209.

Hermes-Lima, M.; Santos, N.C.F.; Alencastro, A.C.R. and Ferreira, S.T.

2007. Whither Latin America? trends and challenges of science in Latin America. IUBMB Life 59, 199-210.
Holmgren, M.; Schnitzer, S.A. and Triendl, R.

2004. Science on the Rise in Developing Countries. PLoS Biology 2, e1. doi:10.1371/journal.pbio.0020001

King, D.A.

2004. The scientific impact of nations. Nature 430, 311-316. doi:10.1038/430311a

De Moya-Anegón, F. and Herrero-Solana, V.

1999. Science in america latina: A comparison of bibliometric and scientific-technical indicators. Scientometrics. 46, 299-320.

RICYT

2017. Red de Indicadores de Ciencia y Tecnología Iberoamericana e Interamericana. Disponible en http://www. ricyt.org/indicadores. Consultado: 15/07/2017.

Santa, S. and Herrero Solana, V.

2010. Producción científica de América Latina y el Caribe: una aproximación a través de los datos de Scopus (19962007). Revista Interamericana de la Biblioteca de Medellín 33, 379-400.

Tahamtan, I.; Safipour Afshar, A. and Ahamdzadeh, K.

2016. Factors affecting number of citations: a comprehensive review of the literature. Scientometrics 107, 1195-1225.

UNESCO

2017. UNESCO Institute for Statistics. Disponible en: http:// uis.unesco.org/. Consultado 04/07/2017.

World Bank Group

2017. World Bank Data. Disponible en: https://data.worldbank. org/indicator. Consultado: 02/07/2017.

Zenteno-Savín, T.; Beleboni, R.O. and Hermes-Lima, M.

2007. The cost of Latin American science. Comparative Biochemistry and Physiology Part A: Molecular \& Integrative Physiology 146, 463-469. 
